\title{
URACO-CISTOPEXIA: NUEVA TÉCNICA DE CISTO-SUSPENSIÓN ASOCIADA AL BURCH PARA EL TRATAMIENTO DEL CISTOCELE E INCONTINENCIA URINARIA DE ESFUERZO, SEGUIMIENTO A LARGO PLAZO
}

\author{
Eduardo Alonso Serrano-Brambila', José Luis Lorenzo-Monterrubio², Virgilio Augusto López- \\ Sámano², Guillermo Montoya-Martínez², Juan Carlos Orozco-Lara ${ }^{3}$ y Guillermo Antonio Ixquiac- \\ Pineda ${ }^{3}$.
}

'Jefe del Servicio de Urología, 2 Urólogos adscritos al Servicio de Urología, ${ }^{3}$ Residente de Urología. Departamento de Urodinamia. Servicio de Urología del Hospital de Especialidades del Centro Médico Nacional Siglo XXI. Instituto Mexicano del Seguro Social. México. Distrito Federal.

\begin{abstract}
Resumen.- OBJETIVO: Comparar la eficacia y morbilidad a largo plazo de pacientes con incontinencia urinaria de esfuerzo a las que se realizó uretro-cervicosuspensión tipo Burch versus el procedimiento de Burch más uraco-cistopexia.
\end{abstract}

MÉTODOS: Se realizó un análisis de cohorte retrospectivo, longitudinal, comparativo y observacional. De enero de 1994 a marzo 2005 se incluyeron 129 pacientes con incontinencia urinaria de esfuerzo (IUE) o incontinencia urinaria mixta (IUM). Cincuenta y cuatro pacientes fueron sometidas a procedimiento de Burch (grupo B) y 75 pacientes a procedimiento de Burch asociado a uraco-cistopexia (grupo BU). La severidad de la incontinencia fue valorada mediante el número de apósitos utilizados durante 24 hrs. La cura se consideró cuando el paciente no requirió ningún dispositivo para la pérdida urinaria; la mejoría como una disminución a una toalla al día y fracaso el uso de más de 1 apósito

\section{CORRESPONDENCIA}

Eduardo Alonso Serrano Brambila Tlacotalpan 59-220 Col. Roma

Delegación Cuauhtémoc.

C.P.06760. (México D.F.)

eserranob@hotmail.com

Trabajo recibido: 26 de septiembre 2007 en 24 horas. En la IUM, el componente de incontinencia urinaria de urgencia (IUU) se evaluó por separado.

RESULTADOS: Se evaluaron 47 pacientes del grupo $B$ y 67 pacientes del grupo BU; a los 12 meses de seguimiento observando una tasa cura/mejoría de la IUE del $74.4 \%$ (29/6) y $97 \%$ (58/7) respectivamente, $p=0.001$ y a los 24 meses de seguimiento 35 pacientes del grupo B y 42 pacientes del grupo BU observando una tasa del $65.7 \%(22 / 3)$ y $97.6 \%$ (37/4) respectivamente, $p=0.014$. La IUM basal se presentó en $53.7 \%$ de pacientes del grupo B y en $58.6 \%$ pacientes del grupo BU. Se analizó en forma aislada la parte de IUU de estas pacientes; a los 12 meses $53.1 \%$ de pacientes del grupo B y $19.4 \%$ del grupo BU presentaban IUU, $p=0.000$ y a los 24 meses $50 \%$ de pacientes del grupo B y $26.19 \%$ del grupo BU presentaban IUU, $p=0.029$. La IUU de novo se presentó en $19.14 \%$ de pacientes de grupo B y en el grupo BU 5.97\%, p=.0000 y a los 24 meses en el grupo B 17.64\% de pacientes y en el grupo BU 13.95\% p=0.005. Las complicaciones relacionadas a la uraco-cistopexia se presentaron en el trans-operatorio y fue apertura vesical en 3 de los primeros casos que se resolvieron con cistorrafia en dos planos y sonda vesical por espacio de 7 días.

CONCLUSIONES: El procedimiento de Burch asociado a la uraco-cistopexia tuvo mayor eficacia en la resolución a largo plazo de la IUE y del componente de IUU que el procedimiento de Burch aislado. La fijación quirúrgica del uraco a la aponeurosis de los rectos anteriores del abdomen mantiene firme la vejiga, lo que probablemente disminuye su desplazamiento durante los esfuerzos, evitando así la tensión en las fijaciones uretro-cervicales, elevando la eficacia del procedimiento de Burch. 
Palabras clave: Uraco-cistopexia. Incontinencia urinaria de esfuerzo. Cistocele.

Summary.- OBJECTIVES: To compare long term efficacy and morbidity in patients with stress urinary incontinence treated using Burch's colpopexy versus Burch's colpopexy plus urachus-cystopexy.

METHODS: Retrospective, longitudinal comparative, observational study in 129 patients with stress urinary incontinence (SUI) or mixed urinary incontinence (MUI). Fifty four patients underwent Burch's colpopexy (group B) and 75 patients underwent Burch's colpopexy and urachus-cystopexy (group B U). These patients completed inclusion criteria from January 1994 to March 2005.

The severity of SUI was evaluated by means of the number of pads used in 24 hours. Cure was defined as patients not using any pad for urinary leakage; improvement, when the number of pads used decreased to one pad a day; and failure when the patients used more than 1 pad in 24 hours. In MUI the urge urinary incontinence (UUI) component was evaluated separately.

RESULTS: After 12 months of follow-up, 47 patients of group $B$ and 67 patients of group $B U$ were evaluated analyzing cure/improvement. Either one were observed in $74.4 \%(29 / 6)$ and $97 \%(58 / 7)$ respectively $(p=$ 0.0011 . At 24 months follow up, in 35 patients of group $B$ and 42 of group BU, a rate of $65.7 \%(22 / 3)$ and $97.6 \%(37 / 4)$ was observed respectively $(p=0.014)$. $\mathrm{MUI}$ was present in $53.7 \%$ of group B and $58.6 \%$ patients of group BU. An independent analysis was made on urge urinary incontinence (UUI) in these patients at 12 months; $53.1 \%$ of group B and $19.4 \%$ of group BU had UUI ( $p=0.000)$. At 24 months, $50 \%$ of patients of group B and $26.19 \%$ of group BU had UUI ( $p=0.029)$. De novo UUI was present in $19.4 \%$ of group $B$ and $5.97 \%$ of group BU ( $p=0.000)$ at 12 months follow-up, and in $17.64 \%$ of patients of group B and $13.95 \%$ of group $B U(p=0.005)$ at 24 months. Complications related to urachus-cystopexy presented trans-operatively: vesical injury in 3 of the initial cases, solved with bladder closure in two layers and vesical catheter for 7 days approximately.

CONCLUSIONS: Burch's procedure in addition to urachus-cystopexy was better for the treatment of SUI and UUI than Burch's procedure alone in a long term clinical follow-up. Surgical fixation of the urachus to the anterior abdominal wall provides extra support to the bladder and probably reduces its displacement during strength, avoiding tension of urethral and bladder neck fixations and increasing the efficacy of Burch's procedure.

Keywords: Urachus-cystopexy. Urinary incontinence. Cystocele.

\section{INTRODUCCIÓN}

La Incontinencia Urinaria de Esfuerzo (IUE) se define como pérdida urinaria involuntaria asociada a situaciones de aumento de la presión intra-abdominal. Se reconocen dos tipos de IUE, el primero por hipermovilidad uretral y el segundo por deficiencia intrínseca del esfínter, sin embargo ambas situaciones con frecuencia se encuentran asociadas (1).

La hipermovilidad de la uretra es una manifestación que resulta de un soporte débil de la vejiga y uretra, mientras que la deficiencia intrínseca del esfínter urinario es una manifestación de un daño estructural $y / o$ funcional.

La Incontinencia Urinaria de Urgencia (IUU) es un síntoma de pérdida urinaria involuntaria asociada a una sensación miccional súbita e imperativa. Usualmente es una manifestación de falta de control de la contracción del músculo detrusor (hiperactividad vesical). La hiperactividad vesical se puede sospechar clínicamente por los síntomas de frecuencia y urgencia.

La Incontinencia Urinaria Mixta (IUM) es la condición de pérdida urinaria involuntaria asociada tanto al esfuerzo como a la urgencia.

La continencia urinaria en términos generales resulta de la interacción recíproca entre las propiedades anatómicas y fisiológicas de la vejiga, uretra, esfínter urinario, piso pélvico y el sistema nervioso como coordinador de estos órganos. La relajación activa de la vejiga en conjunto con la capacidad del esfínter urinario para mantenerse contraído, permite que la orina pueda ser almacenada hasta el momento apropiado en que el vaciamiento vesical se lleve acabo. El papel del piso pélvico y de sus ligamentos es proveer un soporte a la vejiga y uretra, además de permitir la transmisión de la presión abdominal a través de su hiato anterior a la uretra esfinteriana durante los esfuerzos para compensar el aumento de presión intravesical y mantener la continencia. La coordinación realizada por el sistema nervioso central y periférico entre la vejiga, cuello vesical, esfínter uretral y piso pélvico, es crucial para la función de almacenamiento y vaciado urinario. La Incontinencia Urinaria (IU) ocurre cuando existe un desequilibrio de la interrelación entre los componentes del tracto urinario inferior resultado de un daño neurológico, degenerativo o traumático de la pelvis. Se asocia a la edad avanzada, multiparidad, embarazo, parto, obesidad, histerectomía y menopausia (2-3).

La IU es una de las enfermedades más comunes en la mujer, con una prevalencia de $12 \%$ a 
$55 \%$ en todos los grupos de edades. Comparada con otras entidades, la prevalencia de IU es más alta que la hipertensión arterial, depresión y diabetes mellitus (4). También puede producir ansiedad y depresión que afectan las actividades diarias y disminuyen la calidad de vida (5). En los Estados Unidos de Norteamérica se calcula que el costo por este padecimiento es mayor a los $\$ 26$ billones de dólares anuales (6).

Marshall y cols. en 1949 describieron por primera vez la suspensión uretro-vesical retropúbica para el tratamiento de la IUE (7). Burch en 1961 publicó una variante de esta suspensión (8) y desde entonces se han descrito variaciones de cirugías retropúbicas; los puntos básicos son: La suspensión y estabilización de la vagina anterior e indirectamente la base de la vejiga, cuello vesical y uretra proximal (9).

Los procedimientos quirúrgicos para el tratamiento de IUE generalmente ayudan a mejorar el soporte de la unión uretro-vesical, corrigiendo la hipermovilidad uretral. Sin embargo, existe cierta discrepancia respecto al mecanismo preciso por el cual, la continencia mejora con el tratamiento quirúrgico. La preferencia del cirujano, problemas coexistentes, características anatómicas propias del paciente y su salud en general son factores que con frecuencia influyen en la elección del procedimiento quirúrgico.

En un reporte preeliminar (10) con el procedimiento de Burch versus Burch asociado a suspensión vesical, mediante fijación alta del uraco a la aponeurosis de los rectos abdominales (uraco-cistopexia), en mujeres con IUE y cistocele se demostró mejoría estadísticamente significativa en el grado de cistocele entre ambos grupos cuando se realizó la uraco-cistopexia. También se encontró mejoría clínica en la resolución de la IUE, aunque no fue estadísticamente significativa. El objetivo de seguir a largo plazo y aumentar el número de pacientes del estudio fue conocer si el éxito con el procedimiento de Burch asociado a la uraco-cistopexia fue mayor que con el Burch solo.

\section{MATERIALES, PACIENTES Y MÉTODOS}

Llevamos a cabo un estudio de cohorte retrospectivo, longitudinal, comparativo y observacional en mujeres adultas que acudieron al servicio de urología de esta Institución con IUE, asociada - no a IUU. Se realizó historia clínica completa, anamnesis de IU, estudios clínicos, radiológicos y urodinámicos para demostrar datos directos e indirectos asociados a IUE, la cual se demostró objetivamente a través de inspección directa de la pérdida urinaria con maniobra de Valsalva durante la cistometría.

La severidad de la incontinencia fue valorada mediante el número de apósitos utilizados durante $24 \mathrm{hrs}$. La cura se consideró cuando el paciente no requirió ningún dispositivo para el control de la pérdida urinaria; la mejoría como una disminución en el uso de toallas a no más de una toalla al día, siempre y cuando la paciente preoperatoriamente utilizaba más de dos toallas y el fracaso se consideró cuando la paciente usaba dos o más toallas. Para evaluar la morbilidad obstructiva de los procedimientos se utilizó la escala de síntomas de American Urological Association (AUA), Pico de flujo máximo (Qmax) y volumen residual post-miccional (VRPM). Para diferenciar las pacientes con deficiencia intrínseca del esfínter utilizamos presión de cierre uretral máximo (PCUM), longitud uretral funcional (LUF) y área total de cierre uretral (ATCU).

Los criterios de inclusión fueron mujeres adultas con diagnóstico de IUE, asociada o no a IUU, sometidas a procedimiento de Burch solo y Burch asociado a uraco-cistopexia.

Los criterios de no inclusión fueron pacientes con falla previa a cirugía retropúbica o de cabestrillo, con trastornos neurológicos o psiquiátricos que afectaran el tracto urinario inferior, que existiera contraindicación formal para el procedimiento anestésico o quirúrgico y pacientes con factores de riesgo para la recidiva (tosedoras crónicas, deficiencia intrínseca del esfínter, deportistas de alto rendimiento y obesidad grado III).

Los criterios de exclusión fueron pacientes que se perdieron del seguimiento o que cursaran con alguna enfermedad que no permitiera la interpretación adecuada de los resultados.

\section{PROCEDIMIENTOS QUIRÚRGICOS}

Los procedimientos se realizaron bajo bloqueo peridural o anestesia general según valoración por anestesiología. El paciente se colocó en posición decúbito dorsal, instalándose sonda Foley 2 vías, calibre $20 \mathrm{Fr}$ con $10 \mathrm{cc}$ de solución estéril en el globo y se preparó el campo quirúrgico. El abordaje se realizó mediante incisión media infraumbilical o de Pfannenstiel, a preferencia del cirujano. Posteriormente se disecó el espacio de Retzius hasta exponer la pared anterior de la vejiga, la porción intrapélvica de la uretra, la fascia endopélvica lateral y las porciones laterales de la pared vaginal anterior. En ambos grupos de pacientes el procedimiento de uretro-cérvico- 
suspensión fue el de Burch modificado. Se colocaron 3 puntos con ácido poliglicólico del 0 en la fascia endopélvica y pared vaginal anterior a cada lado de la uretra y del cuello vesical, el punto más distal se fijó en la sínfisis del pubis y los puntos más proximales se fijaron al ligamento de Cooper Figura 1 (grupo B).

En el grupo BU se agregó la uraco-cistopexia, la cual se realizó separando el peritoneo de la pared posterior del domo vesical y la porción cefálica a éste (uraco) se fijó mediante una jareta con sutura no absorbible calibre 2 (poliéster trenzado) a la aponeurosis de los músculos rectos abdominales a nivel de la línea media. La tensión de esta fijación fue suave y su altura dependió del tamaño de la vejiga. (Figura $2 \mathrm{~A}$ y $2 \mathrm{~B}$ )

Finalmente se realizó el cierre de la pared y preferentemente no se colocó ningún sistema de drenaje en el espacio de Retzius, a menos que hubiera existido sangrado venoso persistente o apertura vesical. La sonda Foley se retiró alrededor de 48 horas después del procedimiento. Se administró ciprofloxacino $500 \mathrm{mg}$ cada $12 \mathrm{hrs}$ por 7 días en el postoperatorio. La sutura de piel se retiró alrededor del séptimo día del postoperatorio. Se realizó control en el servicio de urodinamia al mes, 3, 6, 12, 24, $36,48,60,72$ y 84 meses, anamnesis de IU, escala de síntomas de la AUA, exploración física, Qmax, VRPM, examen general de orina y urocultivo.

Para el análisis se utilizó el paquete estadístico SPSS. Se evaluó la significancia de la escala de síntomas de la AUA, número de apósitos, Qmax, VRPM, IUE, IUU, examen general de orina y urocultivo, a los 12, 24,36, 48, 60,72 y 84 meses. Se aplicaron las pruebas paramétricas de Friedman y Wilcoxon, considerando significativo un valor de $\mathrm{p}<0.05$.

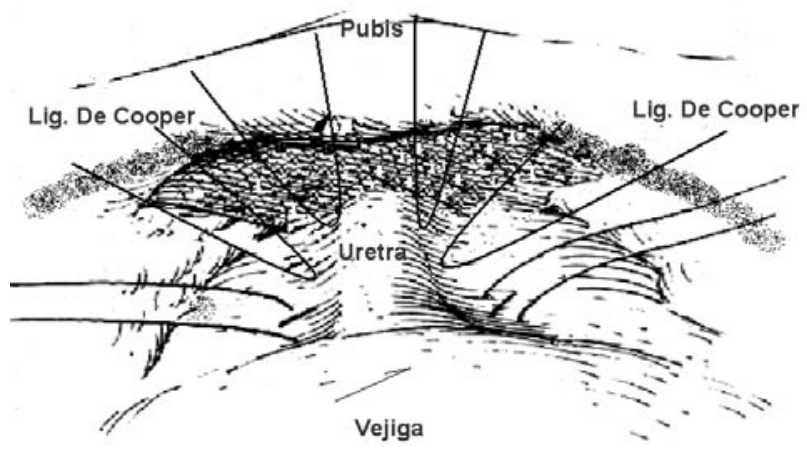

FIGURA 1. Procedimiento de Burch modificado.
Los pacientes firmaron la carta de consentimiento informado para participar en el estudio y publicación de los datos considerando el anonimato de sus nombres.

\section{RESULTADOS}

De enero de 1994 a diciembre del 2004 se estudiaron 69 pacientes con IUE sometidas al procedimiento de Burch de las cuales 54 pacientes fueron incluidas en este estudio (grupo B) y de febrero de 1998 a marzo 2005 se estudiaron 95 pacientes con IUE sometidas al procedimiento de Burch y uracocistopexia de las cuales 75 fueron incluidas en este estudio (grupo BU).

Al analizar la edad, PCUM, LUF, ATCU, histerectomía, menopausia, la escala de síntomas de AUA, Qmax y VRPM no se observaron diferencias estadísticamente significativas entre ambos grupos exceptuando el antecedente de histerectomía que fue mayor para el grupo B (Tablas I y II).

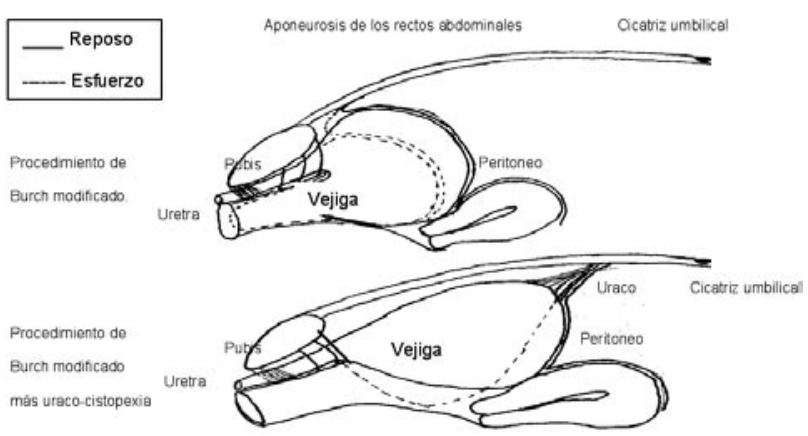

FIGURA 2A. Procedimiento de uraco-cistopexia.

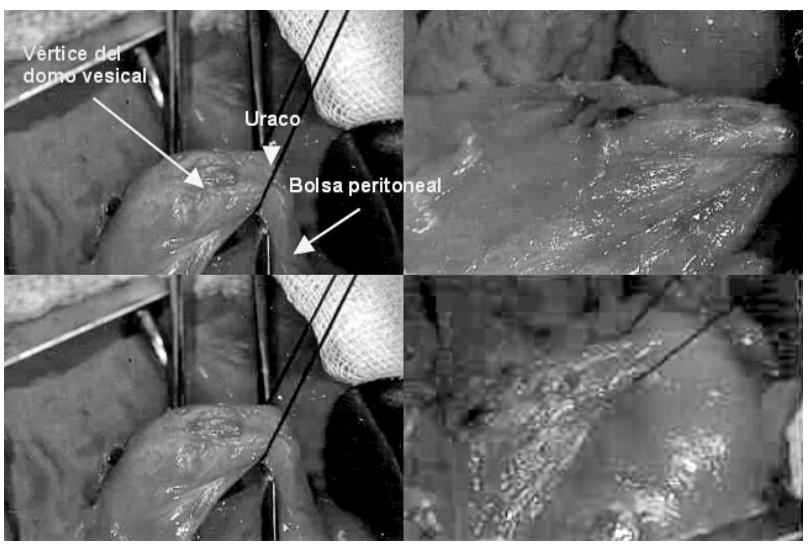

FIGURA 2B. Procedimiento de fijación del uraco a aponeurosis de los músculos rectos abdominales a nivel de línea media. 
TABLA I. EDAD, LONGITUD URETRAL FUNCIONAL (LUF), PRESIÓN DE CIERRE URETRAL MÁXIMO (PCUM), ÁREA TOTAL DE CIERRE URETRAL (ATCU), ANTECEDENTE DE MENOPAUSIA (MEN) E HISTERECTOMÍA (HTA).

\begin{tabular}{|l|c|c|c|c|c|c|}
\hline Grupo & Edad (años) & LUF (mm) & PCUM (cmH2O) & ATCU (mm*cmH2O) & MEN & HTA \\
\hline B & $49(27-73)$ & 30.2 & 72.8 & 1172.4 & 36 & 49 \\
\hline BU & $48(18-74)$ & 28.2 & 75.9 & 1245.7 & 51 & 5 \\
\hline P & $* N S$ & $* N S$ & $* N S$ & $* N S$ & $* N S$ & $* * 0.013$ \\
\hline
\end{tabular}

*Mann Whitney $\quad$ ** $X 2 \quad B=$ Burch $\quad B U=$ Burch + Cisto-uracopexia $\quad$ NS= No significativo

La media basal de toallas utilizadas para control de IUE en el grupo $B$ fue 2.72 y para el grupo BU 2.67. A los 12 meses de seguimiento se evaluó la cura/mejoría de IUE en 47 pacientes en el grupo $\mathrm{B}$ y 67 pacientes del grupo $\mathrm{BU}$, observando una tasa del $74.4 \%(29 / 6)$ y $97 \%(58 / 7)$ respectivamente, $\mathrm{p}=0.001$ y los 24 meses se evaluaron 35 pacientes en el grupo B y 42 pacientes en el grupo BU observando una tasa del $65.7 \%(22 / 3)$ y $97.6 \%(37 / 4)$ respectivamente, $\mathrm{p}=0.014$.

A los 36, 48, 60 meses se observó mayor tasa de cura-mejoría consistentemente en el grupo $\mathrm{BU}$, pero no fue estadísticamente significativa. A los
72 y 84 meses la muestra de pacientes fue demasiado pequeña para el tratamiento estadístico (Tabla III).

La IUU basal se presentó en $53.7 \%$ del grupo $B$ y en $58.6 \%$ del grupo BU. A los 12 meses de seguimiento $53.1 \%$ de pacientes en el grupo $B$ y $19.4 \%$ de pacientes en el grupo BU presentaban IUU $p=0.000$. A los 24 meses $50 \%$ en el grupo B y $26.19 \%$ en el grupo BU de pacientes presentaban IUU $p=0.029$.

A los 36, 48, 60, 72 y 84 meses no hubo diferencia estadísticamente significativa (Tabla III).

TABLA II. RESULTADOS DE LA ESCALA DE SÍNTOMAS DE LA AUA, PICO DE FLUJO MÁXIMO (QMAX) Y VOLUMEN RESIDUAL POSTTMICCIONAL (VRPM).

\begin{tabular}{|c|c|c|c|c|c|c|c|}
\hline & Grupo & Basal & $12 \mathrm{~m}$ & $24 \mathrm{~m}$ & $36 \mathrm{~m}$ & $48 \mathrm{~m}$ & $60 \mathrm{~m}$ \\
& $\mathrm{~B}(\mathrm{~N})$ & 54 & 47 & 35 & 22 & 19 & 15 \\
& $\mathrm{BU}(\mathrm{N})$ & 75 & 67 & 43 & 25 & 17 & 15 \\
\hline \multirow{2}{*}{ AUA (puntos) } & $\mathrm{B}$ & 15.9 & 11.7 & 11.3 & 10.6 & 9.7 & 11.6 \\
\cline { 2 - 8 } & $\mathrm{BU}$ & 14.2 & 9.3 & 10.8 & 9.9 & 12.6 & 8.1 \\
\hline \multirow{2}{*}{$\begin{array}{c}\text { Qmax. } \\
(\mathrm{ml} / \mathrm{seg})\end{array}$} & $\mathrm{B}$ & 28.5 & 28.9 & 30.8 & 24.0 & 23.7 & 26.6 \\
\cline { 2 - 8 } & $\mathrm{BU}$ & 30.4 & 27.6 & 25.3 & 27.5 & 31.5 & 27.8 \\
\hline \multirow{2}{*}{ Ores } & $\mathrm{B}$ & 26.0 & 24.9 & 23.5 & 27.8 & 23.8 & 24.0 \\
\cline { 2 - 8 }$(\mathrm{ml})$ & $\mathrm{BU}$ & 44.9 & 25.0 & 27.6 & 26.0 & 18.7 & 25.2 \\
\hline
\end{tabular}


La IUU de novo se presentó a los 12 meses en $19.14 \%$ de pacientes del grupo $B$ y $5.97 \%$ del grupo $\mathrm{BU}, \mathrm{p}=.000$. A los 24 meses se presentó en $17.64 \%$ del grupo $B$ y en $13.95 \%$ del grupo BU, $\mathrm{p}=0.005$ (Tabla III).

Las complicaciones relacionadas a la uracocistopexia se presentaron en el trans-operatorio y fue apertura vesical en 3 de los primeros casos y se resolvieron con cistorrafia en dos planos y sonda vesical por espacio de 7 días.

\section{DISCUSIÓN}

Aún no está claro el mecanismo intrínseco involucrado en la fuga urinaria a través de la zona de alta presión en la uretra media durante el esfuerzo, sin embargo, consideramos que es fundamental la relación anatómica que guarda el esfínter uretral con el hiato anterior de los músculos del piso pélvico.

Al igual que en los músculos de la pared abdominal hay un incremento de la actividad mioeléctrica en el piso pélvico durante el esfuerzo en forma refleja, transmitiendo la presión generada directamente hacia el esfínter uretral que se encuentra rodeado por el hiato anterior de los músculos eleva- dores del ano. En nuestra percepción del mecanismo fisiopatológico de IUE en la mujer, se debe a que la hipermovilidad uretral en dirección caudal que se presenta durante el esfuerzo, ocasiona una pérdida en la relación entre la musculatura del piso pélvico y el esfínter uretral, dejando a este último sin posibilidad de recibir presión adicional y así compensar el aumento de la presión intra-vesical generada por el esfuerzo, manifestándose de esta forma la pérdida involuntaria de orina.

Es importante resaltar que la inestabilidad de la uretra media donde se encuentra el esfínter uretral en íntima relación con la musculatura pélvica es el punto en el que coinciden la mayoría de las teorías respecto a la etiología de la IUE y el éxito del tratamiento quirúrgico depende del adecuado y permanente re-establecimiento de esta estabilidad.

Exceptuando los cabestrillos, la mayoría de las técnicas quirúrgicas actuales promueven la fijación anterior de los elementos de la fascia endopélvica lateral y pared vaginal anterior a diversas estructuras como el pubis (7), ligamento de Cooper (8), fascia obturatriz (11) y vaina anterior de los rectos abdominales (12-14); técnicas que han tenido porcentajes de éxito que oscilan entre 31 y $97 \%(15-16)$. Estos abordajes producen en mayor

TABLA III. RESULTADOS EN TÉRMINOS DE CURA-MEJORÍA (C/M) DE IUE, IUU E IUU DE NOVO DESPUÉS DEL PROCEDIMIENTO PARA AMBOS GRUPOS.

\begin{tabular}{|c|c|c|c|c|c|c|c|}
\hline & Grupo & $\begin{array}{l}\text { Basal } \\
\text { N (\%) }\end{array}$ & $\begin{array}{c}12 \mathrm{~m} \\
\mathrm{~N}(\%) \mathrm{c} / \mathrm{m}\end{array}$ & $\begin{array}{c}24 \mathrm{~m} \\
\mathrm{~N}(\%) \mathrm{c} / \mathrm{m}\end{array}$ & $\begin{array}{l}36 \mathrm{~m} \\
\mathrm{~N}(\%)\end{array}$ & $\begin{array}{l}48 \text { m } \\
N(\%)\end{array}$ & $\begin{array}{l}60 \mathrm{~m} \\
\mathrm{~N}(\%)\end{array}$ \\
\hline \multirow{3}{*}{ IUE } & B & $54(100)$ & $47(74.4)$ 29/6 & $35(65.7) 22 / 3$ & 22 (77.2) & 20 (80.0) & $15(66.6)$ \\
\hline & $B U$ & 75 (100) & 67 (97.0) 58/7 & $42(97.6) 37 / 4$ & $26(96.1)$ & $17(94.1)$ & 15 (93.3) \\
\hline & & NS & $P=0.001$ & $p=0.014$ & NS & NS & NS \\
\hline \multirow{3}{*}{ IUU } & B & $54(53.7)$ & $47(53.1)$ & $34(50.0)$ & $21(42.8)$ & $20(50.0)$ & $14(50.0)$ \\
\hline & BU & 75 (58.6) & 67 (19.4) & $42(26.1)$ & 25 (36.0) & $17(41.1)$ & 15 (33.3) \\
\hline & & NS & $p=0.000$ & $p=0.029$ & NS & NS & NS \\
\hline \multirow{3}{*}{$\begin{array}{l}\text { IUU de } \\
\text { novo }\end{array}$} & B & - & $47(19.1)$ & $34(17.6)$ & 21 (19.0) & $20(25.0)$ & 14 (13.3) \\
\hline & $\mathrm{BU}$ & - & $67(5.9)$ & $42(13.9)$ & 25 (15.3) & 17 (1 1.7) & 15 (20.0) \\
\hline & & & $p=0.000$ & $p=0.005$ & NS & NS & NS \\
\hline
\end{tabular}


- menor medida algún grado de obstrucción uretral, disminuyendo la eficacia del vaciamiento vesical y requiriendo en algunos casos largos periodos de cateterismo uretral por volúmenes de orina residual elevados (16-17).

Dichos procedimientos quirúrgicos en muchas ocasiones no son suficientes por sí mismos para la corrección del cistocele, especialmente en pacientes con defectos importantes (grado III y IV), por lo que generalmente se utiliza un procedimiento adicional por vía vaginal para su corrección quirúrgica (18).

El planteamiento original que precedió a este estudio se basó en una visión anatómica y funcional que intentaba resolver el problema del cistocele observado después de la uretro-cervico-suspensión retropúbica y con ello posiblemente mejorar el vaciamiento vesical en el postoperatorio al estar la vejiga en una posición más alta. Los resultados de un estudio preliminar (10) demostraron mejoría estadísticamente significativa de la eficacia en la corrección del cistocele cuando se agregó la uraco-cistopexia al procedimiento de Burch, evaluado a los 24 meses de seguimiento. En el mismo estudio también se observó mejoría en la resolución de IUE e IUU pero sin ser estadísticamente significativo, ello quizá por el tamaño pequeño de la muestra (30 pacientes de cada grupo). Esta observación estimuló que continuáramos el seguimiento de estas y de las nuevas pacientes que conforman este informe.

Kiølhede $\mathrm{P}$ y cols. en un estudio para investigar los resultados a largo plazo de la eficacia de la colpo-suspensión de Burch demostraron a través de una encuesta postal realizada con una media de 14 años después del procedimiento, la incontinencia urinaria fue experimentada por el $56 \%$ de los respondedores y solo el $19 \%$ de ellos no reportó episodios de incontinencia, los síntomas de IUE ocurrieron en el $26 \%$, IUU en el $17 \%$ y la incontinencia urinaria mixta en el $16 \%$. En el $15 \%$ de los pacientes los síntomas de IU fueron atípicos y no pudieron ser categorizados (19). En otro estudio Lapitan, MC y cols. en 91 mujeres con IUE en quienes se les realizó la colposuspensión de Burch, reportaron curación en el $71 \%$ de las pacientes cuando la vejiga era estable preoperatoriamente y en $57 \%$ de las pacientes que además tenían hiperactividad del detrusor. Después de 5 años únicamente el $52 \%$ del grupo de estudio estuvo completamente seco y libre de complicaciones; alrededor del 30\% necesitaron terapia posterior para la incontinencia (20).

En una revisión sistemática de varios estudios reportaron tasa de cura de la IUE entre $68.9 \%$ a
$88 \%$ con la colposuspensión retropúbica abierta. La evidencia disponible indica que la colposuspensión retropúbica abierta es una modalidad de tratamiento efectiva, especialmente a largo plazo. Las tasas de continencia global oscilan dentro del primer año entre 85 a $90 \%$ y a los 5 años el $70 \%$ permanecen $\operatorname{secos}(21)$.

No consideramos que la uraco-cistopexia pueda mejorar la eficiencia a largo plazo de las técnicas de cabestrillo, ya que es conocido que la suspensión con cintas suburetrales o sub-trigonales es sumamente fuerte y tienden a mantener su eficacia a través del tiempo, sin embargo en pacientes en las que el cistocele asociado es importante (grado II-IV), puede ser de utilidad para su corrección.

La IUE tuvo una mejoría estadísticamente significativa para el grupo de BU a los 12 y 24 meses. La razón de que solo en estos cortes temporales se haya encontrado diferencia estadísticamente significativa, probablemente se debe al mayor tamaño de la muestra, ya que la diferencia permaneció a los 36 y 60 meses pero el tamaño de la muestra disminuyo y con ello su potencia estadística.

Una posible causa de mayor recurrencia en pacientes con procedimiento de Burch es la presión continua sobre los puntos de fijación uretro-cervicales cuando la vejiga se desplaza hacia abajo durante la micción o durante los esfuerzos, al no estar firmemente suspendida. La fascia endopélvica y pared vaginal anterior que se utilizan en el procedimiento, no permiten asegurar un resultado mejor a largo plazo, debido a que son relativamente débiles para el soporte del tracto urinario inferior, y el éxito de la cirugía más bien depende del grado de fibrosis que se desarrolle entre los tejidos parauretrales y paracervicales a la cara postero-lateral del pubis o de los ligamentos de Cooper en el postoperatorio.

Cuando se agrega la uraco-cistopexia al procedimiento de Burch se evita el desplazamiento de la vejiga durante los esfuerzos y la micción y con ello se evita la tensión sobre los puntos uretro-cervicales y esto aumenta la durabilidad del procedimiento. Es clara la ventaja de los cabestrillos en este sentido, debido a que el sostén uretro-cervical es muy fuerte y no depende de la fibrosis de los tejidos, lo que respalda su mayor eficacia en la resolución a largo plazo de la IUE comparado con el resto de las técnicas. No obstante, su utilización es limitada debido a la mayor morbilidad potencial y riesgo de trastornos del vaciamiento vesical postoperatorio.

La IUU tuvo una mejoría significativa para el grupo de BU a los 12 y 24 meses. Hasta aho- 
ra no tenemos una explicación satisfactoria de estos hallazgos, no obstante podría estar relacionada con la posición más vertical del piso vesical y trígono respecto al eje corporal promoviendo que exista menor presión directa del volumen vesical sobre estas estructuras, especialmente durante la posición erecta disminuyendo la sensibilidad miccional e IUU.

El tiempo quirúrgico utilizado para la realización de la uraco-cistopexia osciló entre 5 y 20 minutos, por lo que no representa un aumento importante del tiempo operatorio. Finalmente consideramos que la uraco-cistopexia es un procedimiento sencillo, de baja morbilidad y que requiere una curva de aprendizaje corta.

\section{CONCLUSIONES}

El procedimiento de Burch asociado a la uraco-cistopexia tuvo mayor eficacia en la resolución a largo plazo de la IUE y del componente de la IUU que el procedimiento de Burch aislado. La fijación quirúrgica del uraco a la aponeurosis de rectos anterior del abdomen, mantiene firme la vejiga, lo que probablemente disminuye su desplazamiento durante los esfuerzos, evitando así la tensión en las fijaciones uretro-cervicales, elevando la eficacia del procedimiento de Burch.

\section{AGRADECIMIENTOS}

Srta. Enf. Amable Alfaro Vázquez y Catalina Cruz Zamora Ruíz, técnicas del servicio de urodinamia por su apoyo en los estudios urodinámicos.

Dr. Edilberto Peña de León, neuropsiquiatra y maestro en ciencias médicas por el apoyo metodológico y estadístico en este trabajo.

Al Dr. Abraham M Santacruz-Romero y al Dr. Efraín Maldonado-Alcaraz por la traducción de este artículo al inglés.

\section{BIBLIOGRAFÍA y LECTURAS RECOMENDADAS (*lectura de interés $y^{* *}$ lectura fundamental)}

1. Kreder KJ. Managing incontinence: one size does not fit all. Contemp Urology 2002; 14 (suppl 9): 548.

2. Sutherland SE. Treatment options for female urinary incontinence. Medical Clinics of North America 2004; 88: 140-67.

3. Burgio KL, Matthews KA, Engel BT. Prevalence, incidence and correlates of urinary incontinence in healthy middle age women. J Urol 1991; 146:1225.

4. Disponible en Heart Association. Heart Disease and Stroke Statistics-2008 Update (on line). Última actualización 2008.<http://www. americanheart.org/downloadable/heart/1200078608862HS_ Stats\%202008. final.pdf > Consulted: November 3rd 2008)

5. Du Beau CE, Kiely KD, Resnick NM. Quality of life impact of urge incontinence in older persons: new measure and conceptual structure. J Am Geriatric Soc. 1999; 47:989-94.

6. Wagner TH. Economic cost of urinary incontinence in 1995. Urology 1998; 51: 335-61.

7. Marshall VF, Marchetti A, Krantz A. The correction of stress incontinence by simple vesico-urethral suspension. Surg Gynecol Obstet 1949; 88: 509.

8. Burch JC. Urethrovaginal fixation to Cooper's ligament for the correction of stress incontinence, cystocele and prolapse. Am J Obstet Gynecol 1961; 81:281.

9. Bates P, Bradley WE, Glenn E. The standardization of terminology of lower urinary tract function. J Urol 1979; 121:551.

10. Serrano B. Uracopexia: Nueva técnica de cisto-suspensión asociada al Burch para el tratamiento del cistocele e incontinencia urinaria de esfuerzo. Bol Col Mex Urol 2006; 21: 44-50.

11. Tanagho E. Urodynamics of female urinary incontinence with emphasis on stress incontinence. J Urol 1979; 122: 200.

12. Mommsen S, Folsdtang A, Elving L, Lam GW. Association between urinary incontinence in women and previous history of surgery. Br J Urol 1993; 72: 30.

13. Weidner AC, Versi E. Physiology of micturition. In: Ostergard R, editor. Urogynecology and Urodynamics: Theory and Practice. New Jersey, NJ: William \& Wilkins; 1996. p.33.

14. De Lancey JO. Structural support of the urethra as it release to stress urinary incontinence: the hammock hypothesis. Am J Obstet Gynecol 1994; 170: 1713.

15. Nygaard IE, Kreder KJ, Lepic MM. Efficacy of pelvic floor muscle exercises in women with stress urinary incontinence. Am J Obstet Gynecol 1996; 174: 120.

16. Mills R. Persat R, Ashken H. Long-term follow up results with the Stamey operation for stress incontinence of urine. Br J Urol 1996; 77: 86.

17. Wall LL, Hewitt JK. Voiding function after Burch colposuspension for stress incontinence. J Reprod Med 1996; 41:161.

18. Raz S, Golomb J, Klutke C. Four comer bladder and urethral suspension of moderate cystocele. J Urol 1989; 142: 712.

19. Kjølhede P. Long-term efficacy of Burch colposuspension: a 14-year follow-up study. Acta Obstet Gynecol Scand. 2005; 84: 767-72.

20. Lapitan MC, Cody DJ, Grant AM. Cochrane Database Syst Rev. 2005 Jul 20: (3) CD002912. Review.

21. Walters M D. Surgical management of stress urinary incontinence: Ostergard R, editor. Urogynecology and Urodynamics: Theory and Practice. New Jersey, NJ: William \& Wilkins, 2004. p. 93-103. 